EOMmun Communication et organisation

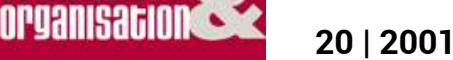

La communication du risque

\title{
Communication, savoir et developpement local
}

\section{Christian Mesnil}

\section{OpenEdition}

Journals

Édition électronique

URL : http://journals.openedition.org/communicationorganisation/2592

DOI : 10.4000/communicationorganisation.2592

ISSN : 1775-3546

\section{Éditeur}

Presses universitaires de Bordeaux

\section{Édition imprimée}

Date de publication : 1 novembre 2001

ISSN : 1168-5549

\section{Référence électronique}

Christian Mesnil, «Communication, savoir et developpement local », Communication et organisation [En ligne], 20 | 2001, mis en ligne le 27 mars 2012, consulté le 04 mai 2019. URL : http://

journals.openedition.org/communicationorganisation/2592; DOI : 10.4000/

communicationorganisation. 2592

Ce document a été généré automatiquement le 4 mai 2019.

(c) Presses universitaires de Bordeaux 


\title{
Communication, savoir et developpement local
}

\author{
Christian Mesnil
}

1 La communication va de soi. Elle passe même pour être un des fondements de l'humanité car c'est à partir du moment où l'échange a pu s'instaurer entre des hominidés que les deux bases de la civilisation: coopération sociale et capitalisation du savoir, étaient établies. La communication a longtemps été considérée comme un effet de mode ou un luxe inutile quand elle n'était pas associée aux bavardages et débats stériles auxquels s'opposait l'efficacité de ceux qui concrétisaient et produisaient. D'aucuns considèrent encore qu'elle ne mérite pas qu'on y consacre autant d'énergie, de réflexion ou d'argent.

2 Au fur et à mesure que se multiplient quantitativement et qualitativement des échanges immatériels à travers la planète, que les connaissances et organisations se complexifient, la lisibilité, la visibilité et l'impact des messages adressés ou reçus deviennent primordiaux. Nombreux sont les sociologues qui, à propos de l'évolution de la société contemporaine, abandonnent l'appellation pourtant récente de société post moderne pour parler désormais de société cognitive, de société du savoir ${ }^{1}$ ou de société de l'information.

3 La présente réflexion tente à la fois d'identifier et de relativiser certains aspects de cette évolution en évoquant successivement le contenu, les intermédiaires, la vitesse, la transparence et la gratuité des transferts d'information. Seront ensuite examinés quelques mutations de la communication des organisations, puis l'individualisation croissante et la recherche d'une parole vraie avant d'évoquer en guise de conclusion le concept de démocratie cognitive.

\section{Des synthèses impossibles... et indispensables}

4 Se faire une opinion sur l'état d'avancement des sciences relève presque aujourd'hui d'une mission impossible. Le chercheur, pour y parvenir dans son propre domaine, doit sans cesse limiter celui-ci et hyperspécialiser ses investigations. "Tout chercheur sait 
bien aujourd'hui qu'il n'y a de travail fécond en science qu'en investissant son énergie dans un domaine particulier, qui apparaît très exigu à celui qui le découvre pour la première fois (...). Le scientifique aujourd'hui est dans un paysage éclaté : le temps des encyclopédistes est terminé. Il n'y a plus guère de synthèse possible pour les diverses sciences particulières. $»^{2}$

Certes le développement des réseaux informatiques facilite la mutualisation des connaissances et renforce l'efficacité de rencontres instantanées entre chercheurs aux extrémités de la planète. Il en résulte la production d'un savoir en direct et en progression démultipliée auquel tout le monde semble avoir accès pour peu qu'il se connecte à un réseau. Pourtant, le fait d'avoir la possibilité matérielle de se brancher sur la toile (dont les utilisateurs ne représentent que $2 \%$ de la population de la planète...) n'implique pas plus que l'ouverture d'un ouvrage ou d'une revue la compréhension de ce qui s'y trouve consigné.

Par ailleurs les conséquences d'une découverte, son maillage dans le temps et dans l'espace avec d'autres savoirs échappe de plus en plus aux scientifiques eux-mêmes. Il semble que les connections soient désormais plus souvent assurées par l'ordinateur dont Joël de Rosnay dit «qu'en rendant la complexité compréhensible et en autorisant les expériences informatiques, il est le catalyseur qui rend possible la synthèse de nouvelles formes de vie. ${ }^{3}$ "Cet emballement d'une science qui nous échappe de plus en plus, dénoncé depuis longtemps par les pessimistes, semble parvenir à un stade où tout le monde s'interroge. Les 4 et 5 septembre 2000, la réunion des comités d'éthique nationaux des quarante et un pays membres du Conseil de l'Europe sur le thème science, communication et société soulignait combien ce qui est techniquement réalisable n'est pas forcément souhaitable: clonage thérapeutique, xénotransplantation (utilisation d'organes animaux pour soigner les hommes) etc. Il insistait sur la nécessité de rendre accessible au plus grand nombre des bases scientifiques...dont les spécialistes eux-mêmes ont grand peine à dresser l'état des lieux exhaustif et actualisé !4

\section{Le passage obligé par des intermédiaires qui facilitent et opacifient}

7 La sociologie des sciences comme l'analyse des discours ont tour à tour étudié les effets de mode, les enjeux en termes de notoriété, de concurrence ou de financement, les stratégies argumentatives de la communication scientifique ${ }^{5}$. Au fur et à mesure que le contact direct et instantané avec le savoir est techniquement facilité, sa quantité et sa complexité rendent indispensables la constitution de relais chargés de rendre sinon transparente, du moins accessible l'explosion actuelle des connaissances.

8 Les médiateurs de l'ombre sont de plus en plus nombreux. Les documentalistes traditionnels ont plus que jamais à transmettre des informations issues d'une veille documentaire indispensable après les avoir indexées (descripteurs) et formatées suivant différents objectifs ou supports. Avec l'utilisation des outils informatiques, de nouveaux métiers deviennent une nécessité pour, par exemple, constituer les banques de données, concevoir de nouveaux produits multimédia ou cartographier les territoires de la toile sans cesse en mouvement.

9 Le statut des enseignants se modifie pour devenir progressivement une sorte d'interface entre les apprenants et différents outils (cd roms, Internet, hypertextes) conçus par les 
industries éducatives pour que « les données nécessaires soient au bout des doigts et de plus en plus souvent exemplifiées ${ }^{6} »$. Des salles en accès libres, des espaces scientifiques de loisir (Cité des sciences de la Villette ou Futuroscope de Poitiers) proposent des vulgarisations ludiques d'un savoir modélisé ou mis en spectacle par des concepteurs anonymes.

Des stratégies de veille, de validation, de sélection des informations sont également mises en place par les médias, scientifiques ou non. Vulgariser sans simplifier abusivement ni avoir de priorités partisanes liées aux écoles de pensée, au financement du journal ou de la recherche relatée n'est pas simple. De plus «les scientifiques recherchent la connaissance, la société en demande les bénéfices. Le journaliste scientifique est trop souvent en position de vendeur de la science et donne de faux espoirs à la société ellemême avide de certitudes. $\aleph^{7}$

11 Enfin, ultimes intermédiaires et non des moindres, figure la caste des experts dont l'arrogance et l'obscurantisme sont de plus en plus régulièrement (injustement?) stigmatisés ainsi que leur incapacité «à concevoir ce qui se trouve hors de leur compétence spécialisée (...) ou à penser les problèmes globaux ou fondamentaux. $»^{8}$

Chacun sait combien le filtrage d'un certain nombre d'intermédiaires risque d'altérer le message initial. Mais le citoyen n'a pas d'autre choix possible que de passer par ces médiations qu'il a intérêt à varier comme des éclairages ou des angles de prise de vue pour en limiter l'opacité ou la partialité.

\section{Accélération de la vitesse de circulation des informations et incompressibilité des temps d'assimilation}

13 Dans La refondation du monde, Jean Claude Guillebaud observe que nous nous laissons entrainer dans « une course paradoxale qui ne sait plus où elle va mais y va de plus en plus vite, dans la frénésie d'une cavalcade angoissée et déshumanisante (...) nous ne sommes plus portés par une représentation du futur mais emportés par une impatience obligatoire. ${ }^{9}$ " Cette course perpétuelle épargne de moins en moins de modes de vie, de zones géographiques ou de catégories socio professionnelles. Or il est des domaines comme celui de l'apprentissage ou de la recherche où le temps est difficilement compressible.

Dans un univers envahi par les médias, l'éphémère et la nouveauté, arrêter de zapper, procéder à des arrêts sur image voire à des retours en arrière, interrompre le flux continu de discours en ligne, en temps réel ou en boucle devient à la fois de plus en plus difficile et nécessaire. Pouvoir traiter, évaluer, stocker, compacter ou rejeter l'information brute pour la transformer en savoir, puis mettre ce dernier en lien avec d'autres connaissances afin d'établir de nouvelles connections, de façonner des convictions au moins un peu personnalisées nécessite une maîtrise du temps qui nous échappe.

En lien avec de nombreux spécialistes, Alain Cordier a été chargé d'établir un rapport sur l'évolution du livre et de l'édition dans le cadre d'un programme gouvernemental d'action intitulé «Préparer l'entrée de la France dans la société de l'information ». Il a souligné en particulier l'importance de la temporalité. "Il faut s'interroger sur la compatibilité entre la lutte constante pour le gain de temps que représente le 
développement des technologies numériques, et le nécessaire usage de la durée que réclame l'apprentissage des connaissances et au-delà, de la pensée elle-même. La durée s'oppose à l'immédiateté et fait droit à l'humanité de chacun qui ne peut se construire que dans le temps et non pas hors de lui, dans l'instant. $»^{10}$

Ce devoir qui incombe à tout citoyen devient plus impérieux encore pour les chercheurs à l'origine de cette explosion et accélération du savoir. L'intuition et la fulgurance ne peuvent devenir le processus dominant voire exclusif. Le «science sans conscience n'est que ruine de l'homme » des humanistes devient une absolue nécessité étant données les compétitions en cours et les conséquences possibles pour la planète. "Le véritable scientifique est celui qui sait arrêter son travail pour réfléchir à la fin qu'il poursuit. La connaissance n'est pas le fruit obligé du travail, il y faut le génie, le temps de l'intelligence et de l'esprit ${ }^{11}$ » Pourtant, cette maîtrise de la temporalité est souvent un vœux pieux, d'autant que les conséquences, même modélisées de façon virtuelle par l'informatique, restent imprévisibles et que le savoir validé expérimentalement a vocation à être théorisé tôt ou tard quels que soient les mobiles des acteurs...

\section{Transparence et gratuité ou marchandisation?}

La valeur d'une connaissance semble de plus en plus liée à sa nouveauté et sa validité peut se révéler très courte, menacée à tout instant d'obsolescence par l'accélération de la production scientifique d'une extrémité à l'autre de la planète. Par ailleurs les nouvelles technologies permettent un contrôle de plus en plus poussé de l'information. Des compteurs de visite enregistrent l'historique des heures de consultation. Les analyseurs déchiffrent l'origine codée des pays et des serveurs connectés. Il est possible de savoir les autres centres d'intérêt du visiteur, de référencer sur son site d'autres sites qui font de même avec le vôtre. Entre une communication participante de plus en plus horizontale observée par Bernard Miège dès les années $90^{12}$ et le maillage cher à Michel Serre, une vigilance accrue est demandée aux usagers de la toile comme à chaque citoyen qui, même lorsqu'il n'est pas connecté, doit en assumer des conséquences indirectes, (commerce électronique, délinquance, espionnage...)

12 février 2001 à Paris comme dans les cinq autres grandes capitales concernées, le consortium public Hugo (Human Genorme Organisation) a été contraint d'annoncer trois ans avant son terme prévisible les résultats actuels de sa recherche sur le séquençage du génome humain. Pourquoi ? Pour éviter d'être devancé par un concurrent privé, Celera Genomics, aux méthodes contestables (pillage des travaux de Hugo sur Internet selon son directeur pour la France, le professeur Jean Weissenbach). Devant les pistes vertigineuses qui ouvrent une "nouvelle ère thérapeutique ", (expression du ministre français de la recherche M. Schwartzenberg), les enjeux s'avèrent considérables. Le débat en Europe sur la brevetabilité des gènes est significatif de la marchandisation du savoir et de la concurrence internationale impitoyable en ce domaine.

Le pouvoir et la richesse sont de plus en plus concentrés entre les mains de ceux qui sont les plus rapides à produire, à capter ou diffuser un savoir. Pour des raisons politiques, idéologiques, militaires, économiques, toutes les informations ne circulent pas aussi librement que pourrait le laisser croire l'avènement de la société de l'information. Même si les sites gratuits restent les plus nombreux sur le web ( $86 \%$ ), les sites marchands sont en moyenne six fois plus consultés et représentent la moitié de l'audience ${ }^{13}$. L'authentique désintéressement de scientifiques attachés à percer les secrets de la 
matière ou de la maladie, en constante liaison au sein d'une communauté de chercheurs, les discours optimistes sur l'accès, le partage et la gratuité du savoir se heurtent à la réalité des contraintes économiques et des lois du marché.

L'importance des coûts fixes, l'investissement considérable que représente la recherche, la nécessité de produire et de vendre en nombre, le maintien de fait des entreprises les plus aptes à survivre à la concurrence aboutissent à des conséquences contrastées. Certes la bataille des encyclopédies Encarta contre Encyclopaedica Britanica a conduit à l'effondrement des prix publics de leurs CD-roms mais par ailleurs, le coût d'un traitement efficace contre le sida reste prohibitif dans les pays les plus atteints par la maladie. En mars 2001 a débuté en Afrique du Sud un procès inédit. D'un côté l'Association des industries pharmaceutiques suite à la plainte de la multinationale Hoffmann La Roche, de l'autre l'État Sud Africain au motif qu'il autorise les importations parallèles de médicaments génériques en passant outre à l'accord de l'OMC (organisation mondiale du commerce) sur la propriété intellectuelle.

Dans L'économie de la connaissance, Dominique Feray nous montre que le juste milieu entre la rémunération du découvreur, le retour sur investissement des financeurs et le partage légitime d'un patrimoine immatériel commun à l'humanité évolue sans cesse au rythme des créations ${ }^{14}$. La priorité au meilleur, au plus performant, au plus offrant, les brevets, les rentes de situation, l'espionnage scientifique appellent à nuancer singulièrement l'optimisme des tenants de la transparence et de la gratuité dans un espace de partage, coopération et fusion des esprits sans conflit ni critique dont le philosophe Pierre Lévy se fait entre autres, l'interprète dans son ouvrage World philosophie $e^{15}$.

\section{Mutations dans la communication des organisations}

L'appropriation de l'information scientifique et technique entraîne la transformation d'un certain nombre d'usages qui n'est pas sans conséquence sur les organisations et leur communication. La spécialisation évoquée antérieurement implique un souci constant de communiquer pour vulgariser et réintégrer dans des ensembles si l'on veut éviter une segmentation excessive voire incohérente. Par ailleurs les métiers et les fonctions se recomposent autour de projets ponctuels qui nécessitent de se former à de nouvelles compétences. Des échanges différents, peut-être plus solidaires et opérationnels, s'organisent autour d'objectifs précis, alimentés en temps réel par des informations sur les résultats, les performances ou la concurrence.

Dans d'autres cas, au lieu d'un réaménagement de l'organisation, on assiste à sa désintégration matérielle puisqu'elle ne produit plus mais soustraite ou délocalise tout en assurant l'ingénierie. Là encore des conséquences en termes de communication des organisations sont sensibles au moins à deux niveaux. D'une part une certaine prise de conscience de la vulnérabilité et de l'insécurité entraînées par la concurrence, les flux tendus ou la mondialisation nécessite de gérer de façon de plus en plus rationnelle les ressources humaines ${ }^{16}$. D'autre part la coordination technique implique d'être constamment connecté sur des réseaux multiples pour réguler des productions, du stockage ou des flux financiers.

Certes, de nombreuses structures évoluent peu ou ne sont pas elles-mêmes exemptes de dérives et de dysfonctionnements. Des organisations locales favorisent le corporatisme, le clientélisme ou le sectarisme, engendrant des crispations ou des blocages. Il arrive que les 
frais de fonctionnement soient inversement proportionnels à l'efficacité. L'état providence transforme parfois les citoyens en assistés. Il peut se dédouaner facilement de ses responsabilités en pratiquant la langue de bois (épidémies, mondialisation, aléas climatiques). Les organismes internationaux communiquent sur des programmes d'austérité ou une concurrence systématique des individus sans écouter vraiment certains partenaires.

Aucun système humain ne peut prétendre à une validité permanente mais des réseaux de vigilance, des instances de concertation, des processus de partage de l'information et des décisions semblent plus que jamais nécessaires. Le maintien ou le développement d'échanges institutionnalisés, de concertation passe pour incontournable tant au niveau planétaire que pour les organisations intermédiaires. Peu à peu émerge le souci de préserver des groupes locaux même s'ils sont faibles, peu efficaces, non rentables, mal irrigués par les nouvelles technologies. Il importe non de se substituer à eux ou d'en extraire les forces vives qui s'y maintiennent mais de les aider dans un constant dialogue à préserver leur vocation.

S'approprier les évolutions en cours, réfléchir aux moyens et aux fins, peser sur les choix et priorités nécessite des pratiques régulières de coopération, d'échanges, de dialogues collectifs. Étant donné l'accélération du " progrès » et sa complexité, la communication citoyenne devient impérieuse et passe par des relations longues, sociales et institutionnelles. Cela nécessite à différents niveaux, l'engagement individuel dans des instances de concertation aussi imparfaites qu'elles puissent être.

\section{Individualisation croissante et recherche d'une parole vraie.}

Pourtant un phénomène parallèle semble être observé par de nombreux sociologues. Dans son dernier ouvrage ${ }^{17}$, Jean Claude Kaufmann décrit le mouvement continu de l'humanité vers une individualisation croissante. Or, l'aspiration de chacun, non à l'égoïsme ou au repli sur soi mais à la prise en compte de sa spécificité et de ses aspirations se produit dans un contexte particulier. En effet, nous assistons simultanément à la fin d'une société statique où la place de chacun était stable et évoluait peu.

L'environnement actuel plus créatif, évolutif, compétitif ajoute inévitablement au " progrès » des dérives, accidents, déséquilibres ou absurdités. La nature, la masse et la vitesse évoquées antérieurement à propos de l'information rendent sa transparence et sa lisibilité très inégales. La demande de connaissance augmente à cause d'une aspiration légitime à plus de lucidité, d'autonomie et de choix. Mais dans le même temps, en dépit des nouvelles performances technologiques, les informations n'ont jamais été aussi éphémères et fragmentées avec les risques d'asphyxie, de brouillage ou manipulation que l'on sait.

29 En dépit de la rhétorique et de l'art oratoire, l'essentiel ne passe pas toujours par les mots et différents chercheurs étudient la mise en spectacle (décors, costume, éclairage, vedettes) de l'information ${ }^{18}$. Au devoir de vigilance envers une parole construite consciemment ou non pour séduire, émouvoir ou distraire, Jacques Ellul ajoutait deux aspects déterminants et peut-être un peu paradoxaux de la crédibilité d'un locuteur. « Si la personne est crédible, alors on peut croire ses paroles. Nous savons combien 
maintenant sont dévalués les discours de tant d'intellectuels, de tant de politiciens parce que nous avons expérimenté de cent façons qu'ils ne sont pas crédibles, que rien dans leur vie ne correspond à leur annonce. Alors ce sont paroles de vent ». Mais il poursuivait un peu plus loin: "Vous savez bien que jamais la crédibilité d'un homme n'a pu se démontrer scientifiquement. Vous pouvez accumuler les preuves pour ou contre, vous ne déclencherez pas la croyance en une personne. Vous le sentez ou non. Vous croyez quelqu'un parce que c'était lui, c'était moi, dernière limite de la sagesse des relations inter-humaines ${ }^{19}$. »

30 A l'augmentation de la demande individuelle d'information qui s'accompagne paradoxalement d'une vulnérabilité accrue s'ajoute un besoin de communication interpersonnelle renforcée. Les structures, les institutions, les relais, les technologies aussi incontournables qu'ils deviennent ne doivent pas faire écran à l'humain, au contact direct et au non-verbal ${ }^{20}$. Le développement local semble correspondre à un désir croissant d'agir ici et maintenant, de prendre en main son propre destin au sein d'une communauté familière avec laquelle les interactions sont facilitées par la proximité.

\section{Le concept de démocratie cognitive est-il réaliste ?}

"L'ère de la production des objets industriels est close au bénéfice de l'échange immatériel d'information. Désormais grâce aux réseaux et à l'intégration numérique s'instaurent l'économie et la société de l'information. Les savoirs deviennent la matière première de la société et de l'industrie, dans la mesure où ils vont circuler de façon transparente dans le monde entier et permettre la démocratie cognitive ${ }^{21}$. Peut-être que les optimistes de la société du savoir évoqués par Yves Jeanneret dans un ouvrage récent vont un peu vite en besogne.

La transparence de l'information constitue un défi permanent lorsqu'il y a hyper spécialisation, nécessité de synthèses et d'intermédiaires, le tout sur un rythme accéléré. La circulation du savoir reste très dépendante du pouvoir de l'argent. Quant à la démocratie, elle implique de croiser deux logiques, celle d'une communication des organisations participative qui motive ou informe vraiment et celle d'une communication interpersonnelle directe qui favorise un développement local harmonieux.

Cette notion de démocratie cognitive a été mise en avant notamment par Edgar Morin voici plus de dix ans pour contrecarrer « la techno science, l'hypertrophie des états, leurs conséquences conjointes dans la technocratisation, la bureaucratisation, l'hyper spécialisation généralisée comme dans la parcellisation des existences, l'atomisation des individus, les dégradations écologiques et morales qu'elles entraînent ${ }^{22}$. » Au-delà de cette sombre analyse contextuelle, la persistance du concept de démocratie cognitive est peutêtre le signe que, pour le moment, il n'existe pas d'autre orientation en faveur du progrès et du développement.

Certains désertent pour devenir blasés, indifférents, pessimistes, voire cyniques. D'autres critiquent sans examiner les réponses que les acteurs inventent ou pourraient inventer. Mais même si les projets sont aléatoires et les réseaux fragiles, il existe des citoyens qui informent et mobilisent la conscience des individus et des organisations. Le croisement entre différents niveaux de regroupements et d'échanges humains paraît déterminant pour la connaissance, la réflexion, la dynamisation, le contrôle mutuel des acteurs. Les initiatives précises sur le terrain, appelées à durer et s'amplifier, en dépendent et sont 
indispensables au développement local. Cette démocratie cognitive ne deviendra réalité que si émerge une communication sociotechnique sans cesse à réguler pour ne parler ni en théoricien abstrait, ni en empiriste aveugle, ni en militant manipulateur.

\section{BIBLIOGRAPHIE}

BURSEAUX, E., La réflexion citoyenne sur les choix éthiques? In Le Monde, 12 sept.2000.

CORDIER, A., Le livre numérique, Internet et la pensée. In Communication et langage, Retz Paris, N - $22.4^{\mathrm{e}}$ trimestre 1999.

DETERRE, P., De la recherche scientifique. In M.S. vol. 10. Juillet 1994. DUCCINI, H., La télévision et ses mises en scène. Nathan Université 1998. ELLUL, J., La foi au prix du doute. Seuil. 1988.

FERAY, D., L'économie de la connaissance. Edit. La découverte. GENSOLLEN, M., La création de valeur sur Internet. In Reseaux, vol. XVII, n 97, 1999.

GUILLEBAUD, J C, La refondation du monde. Paris : Le seuil, 1999.

HOTIER, H. (sous la direction de), Non verbal et organisation. l'Harmattan 2000.

JEANNERET, Y., Y a t il vraiment des technologies de l'information ? Presse du septentrion 2001.

KAUFMANN, J C, Ego pour une sociologie de l'individu. Nathan 2001. LATOUR, La vie de laboratoire. La production de faits scientifiques. La Découverte, 1988.

LEVY, P., World philosophie. Odile Jacob 2000.

MIEGE, B., La société conquise par la communication. Grenoble : PUG, 1989.

MORIN, E., Le nouveau combat de la laïcité. In Le débat. Gallimard n 58. Janv. Fév 1990.

ROSNAY, J. (de), L'homme symbiotique. Seuil 1995.

TARDIF, J., Intégrer les nouvelles technologies de l'information : quel cadre pédagogique ? E.S.F., 1998.

\section{NOTES}

1. Cf. Forum. Vers une société du savoir prévu à Paris les 31 mai et $1^{\mathrm{er}}$ juin 2001.

2. Deterre Philippe, De la recherche scientifique. In M.S. vol. 10 juillet 94. P.704.

3. De Rosnay Joël, L 'homme symbiotique. Seuil 95. P.92.

4. Burseaux Elisabeth, La réflexion citoyenne sur les choix éthiques? In Le Monde, 12 sept. 2000.

5. Latour et Woolgan, La vie de laboratoire. La production de faits scientifiques. La Découverte, 1988.

6. Tardif Jacques. Intégrer les nouvelles technologies de l'information: quel cadre pédagogique? E.S.F., 1998

7. Moore Heward, directeur de l'Unité pour le partenariat en science cité par Burseaux Elisabeth. In La réflexion citoyenne sur les choix éthiques? op.cit.

8. Morin Edgar, Le nouveau combat de la laïcité. In Le débat. Gallimard n58. Janv. Fév 90. p. 40.

9. Guillebaud Jean Claude, La refondation du monde. Le Seuil, Paris, 1999 p. 120. 
10. Cordier Alain, Le livre numérique, Internet et la pensée. In Communication et langage. Retz Paris. N²2. $4^{\mathrm{e}}$ trimestre 1999. p. 14.

11. Déterre Philippe, De la recherche scientifique. Op. ect p. 706.

12. Miège Bernard, La société conquise par la communication. PUG, Grenoble 1989.

13. Gensollen Michel, La création de valeur sur internet. In Reseaux, vol. XVII, $n^{\circ}$ 97, 1999.

14. Feray Dominique, $L$ 'économie de la connaissance. Edit. La découverte.

15. Levy Pierre, World philosophie. Odile Jacob 2000.

16. Cf. Par exemple, l'apparition des " call centers ".

17. Kaufmann Jean Claude, Ego pour une sociologie de l'individu. Nathan 2001

18. Cf. Duccini Hélène, La télévision et ses mises en scène. Nathan Université 1998.

19. Ellul Jacques, La foi au prix du doute. Seuil. 1988. p. 211 et 213.

20. Hotier Hugues (sous la direction de), Non verbal et organisation. l'Harmattan 2000.

21. Jeanneret Yves, Y a t il vraiment des technologies de l'information? Presse du septentrion 2001.

22. Morin Edgar, Le nouveau combat de la laïcité. In Le débat, Paris : Gallimard n 58 janvier février 1990, p. 40.

\section{RÉSUMÉS}

La communication du savoir est confrontée à un nombre croissant de contradictions. Elles concernent la synthèse de données de plus en plus complexes, l'accès direct aux informations et les intermédiaires pourtant incontournables, la relation au temps à la fois accélérée et incompressible ainsi que la transparence et la gratuité qui sont relatifs. Il en résulte d'une part la nécessité d'une organisation technique et sociale accrue, d'autre part le développement de l'individualisation et du local. De ce fait le concept de démocratie cognitive semble appelé à se renforcer pour accompagner l'évolution des processus de communication.

The communication of knowledge faces a growing number of contradictions. These deal with synthesizing increasingly more complex data, direct access to information and yet the unavoidable existence of intermediaries, a relation to time which is both accelerated and incompressible, as well as a very relative transparency and gratuity. As a result there is, on the one hand, a need for an increasingly technical and social organisation, and on the other hand, a development of individualisation and local concerns. Therefore, the concept of cognitive democracy seems likely to be reinforced so as to accompany the evolution of the communication process.

\section{INDEX}

Mots-clés : savoir, accès, transparence, gratuité, démocratie cognitive 


\section{AUTEUR}

\section{CHRISTIAN MESNIL}

Christian Mesnil est maître de conférences en sciences de l'information et de la communication à l'Université du Littoral Côte d'Opale (Calais). 